\title{
Total Reconstruction of the Afferent Loop for Treatment of Radiation-Induced Afferent Loop Obstruction with Segmental Involvement after Pancreaticoduodenectomy with Roux-en-Y Reconstruction
}

\author{
Konstantinos Blouhos Konstantinos A. Boulas Ilias I. Salpigktidis \\ Anna Konstantinidou Konstantinos Ioannidis Anestis Hatzigeorgiadis \\ Department of General Surgery, General Hospital of Drama, Drama, Greece
}

\section{Key Words}

Pancreaticoduodenectomy $\cdot$ Roux-en-Y reconstruction $\cdot$ Radiation enteritis · Afferent loop obstruction · Surgery

\begin{abstract}
As the literature on afferent loop obstruction (ALO) after pancreaticoduodenectomy (PD) is very limited, standardized rules for its management do not exist. Herein, we report the case of a 65-year-old male patient with chronic ALO who had undergone PD with single Roux-en$Y$ limb reconstruction and adjuvant chemoradiation therapy for pancreatic head adenocarcinoma 2 years earlier. The patient was brought to the operating room with the diagnosis of radiation enteritis of the afferent loop with segmental involvement and concurrent hepaticojejunostomy $(\mathrm{HJ})$ and pancreaticojejunostomy $(\mathrm{PJ})$ stricture. Complete mobilization of the afferent loop, removal of the affected segment and reconstruction were performed. Reconstruction of the afferent loop was a one-way option for the surgeons because the Roux-en-Y reconstruction limited endoscopic access to the afferent loop, and the segmental radiation injury of the afferent loop ruled out bypass surgery. However, mobilization of the affected segment through a field of dense adhesions and revision of the $\mathrm{HJ}$ and PJ were technically demanding.




\section{Introduction}

Afferent loop obstruction (ALO) is a rare complication of pancreaticoduodenectomy (PD), with a reported incidence varying from 1.2 to 13\% [1]. Pannala et al. [2], in their retrospective cohort study, reported 24 (13\%) patients who developed ALO among 186 pancreatic cancer patients treated with PD; in 9 (38\%) patients, obstruction was caused by radiation enteritis of the afferent loop. As ALO is an infrequent complication, much of the current knowledge on management is derived from the accrual of single-surgeon or singleinstitution series. Herein, we present a case of post-PD radiation-induced ALO and concurrent hepaticojejunostomy (HJ) and pancreaticojejunostomy (PJ) stricture. When endoscopic assessment of the afferent loop is limited, such as in cases of Roux-en-Y reconstruction, and segmental involvement of the afferent loop rules out bypass surgery, the authors endorse reconstruction of the afferent loop as a feasible management option.

\section{Case Report}

A 65-year-old man was referred to our hospital due to recurrent episodes of crampy periumbilical pain and associated episodes of emesis over the previous 2 months. The patient had undergone PD 2 years earlier in our surgical department. The initial operation comprised PD along with distal gastrectomy, complete omentectomy, extended lymphadenectomy and Roux-en-Y reconstruction for a moderate differentiated, pT2pN1(2/39)MO, $\mathrm{ly}(+), \mathrm{v}(+), \mathrm{R} 0$ pancreatic ductal adenocarcinoma of the head. After the operation, the patient received concomitant flat infusional 5 -FU chemotherapy $\left(300 \mathrm{mg} / \mathrm{m}^{2} /\right.$ day, 5 days/week) during the entire course of external beam irradiation (50.4 Gy in 33 fractions over 7 weeks). No recurrence was found at the follow-up visits (assessment of CA19.9 levels every 3 months and abdominal CT scan every 6 months).

Abdominal examination was remarkable for tenderness in the right upper quadrant. The laboratory workup revealed a normal white blood cell count, transaminases and slight elevation of bilirubin, alkaline phosphatase, $\gamma$-glutamyl transpeptidase and amylase. Plain abdominal X-rays were normal. Multidetector CT (MDCT) revealed: (a) segmental afferent loop wall thickening and lumen narrowing; (b) absence of lymphadenopathy, and (c) dilatation of the hepatic duct $(9 \mathrm{~mm})$ and the pancreatic duct $(2.9 \mathrm{~mm})$. Ductal pathology was further assessed though magnetic resonance cholangiopancreatography (MRCP) examination which revealed a fixed filling defect at the $\mathrm{HJ}$ and PJ with associated postobstructive hepatic and pancreatic duct dilation.

The diagnosis of chronic ALO along with $\mathrm{HJ}$ and PJ stricture was established; radiation enteritis of the afferent loop and/or the overlap of radiation enteritis and local recurrence in the length of involvement were presumed to be the causes of the obstruction. Elective laparotomy was performed via an extended upper midline incision. Once adhesions from the previous surgery were taken down and the free peritoneal cavity was entered, the liver, hepatoduodenal ligament, stomach, efferent loop, jejunojejunostomy and afferent loop were recognized (fig. 1). The gastric remnant, efferent loop and afferent loop were released from adhesions and fully mobilized. Copious dissection in a field of dense adhesions and scar tissue on either side of the afferent loop enabled recognition of the HJ, PJ and pancreatic body. Peripherally, the afferent loop was transected distal to the involved segment (fig. 2a). Proximally, mobilization of the afferent loop exposed the surgical bed of the previous PD; no macroscopic evidence of local recurrence in the pancreatic bed, the liver or the peritoneal surface was observed (fig. 2b). The HJ was disconnected and a new end-to-side HJ was 
performed. Moreover, the PJ was taken down and examined. A short segment $(1.5 \mathrm{~cm})$ of the remnant pancreas was resected until the dilated pancreatic duct was revealed and a revised duct-to-mucosa PJ was performed using an anastomotic stent (fig. 3). The distal remnant of the afferent loop, which was pulled toward the pancreas through the transverse mesocolon, had a sufficient redundancy to provide construction of the anastomoses without tension. A closed suction drain was placed at the subhepatic space near the anastomoses, and the abdomen was closed with a running No. 1 looped nonabsorbable suture and staples. Histology of the surgical specimen revealed microscopic findings of radiation enteritis without evidence of malignancy. The postoperative course was uneventful, and the patient was discharged home on postoperative day 9. At a follow-up time of 11 months, the patient was alive and asymptomatic; MRCP revealed no evidence of stenosis or diminished flow across the revised anastomoses.

\section{Discussion}

As radiation-induced ALO is an infrequent complication of PD and adjuvant radiotherapy, the literature on management of this complication is limited [3]. Undoubtedly, endoscopy plays a principal role in the management of radiation-induced ALO. However, a Roux-en-Y reconstruction compared to conventional loop reconstruction limits endoscopic access to the afferent loop [4]. Moreover, percutaneous approaches for endoluminal stenting have only been reported in the literature as isolated cases [5]. Consequently, in the setting of PD with Roux-en-Y reconstruction, the afferent loop, left inaccessible after endoscopy, can only be assessed by surgery.

Reoperative surgery after PD is a difficult undertaking; reoperation rates vary from 4 to $11 \%$ among small-volume series [6]. The literature on post-PD reoperation for ALO is very limited. Before making the decision for surgical treatment, the extent of radiation injury of the afferent loop and the patency of the primary $\mathrm{HJ}$ and PJ should be thoroughly evaluated through the use of MDCT and MRCP or secretin-enhanced MRCP. Radiation enteritis typically presents as a segmental area of bowel wall thickening. However, even when all parameters of bowel wall thickening, such as pattern of attenuation, degree of thickening, symmetric versus asymmetric thickening, focal or segmental involvement and associated perienteric abnormalities are assessed, discrimination of the malignant or benign nature of the obstruction is difficult [7]. Because in the length of involvement overlap of radiation enteritis and local recurrence cannot be excluded, mobilization and removal of the affected segment seems to be a reasonable approach. Moreover, as the involvement of the afferent loop is segmental, bypass surgery cannot be performed because there is no normal bowel to perform an anastomosis. Regarding the patency of the primary anastomoses, when evidence of $\mathrm{HJ}$ stenosis is present, endoscopic or radiographic dilatation is successful in most cases [8]. However, endoscopic management of PJ stricture has not been well examined. When evidence of PJ stenosis or radiation injury of the afferent loop around the PJ is present, surgery including takedown/revision of the PJ and/or a modified Puestow procedure should be performed based on the size of the pancreatic duct and degree of pancreatic fibrosis [9].

Taking this background into account, reconstructive surgery seems to be the principal treatment for radiation-induced ALO with segmental involvement after PD with Roux-en-Y reconstruction. Reconstruction of the obstructed afferent loop is generally considered to be a more invasive operation, whereas a smaller operation, such as bypass surgery, is adequate, less dangerous and more reasonable. However, reconstruction should be considered as a therapeutic option in the following two circumstances. Firstly, if the obstructed loop is 
Blouhos et al.: Total Reconstruction of the Afferent Loop for Treatment of RadiationInduced Afferent Loop Obstruction with Segmental Involvement after Pancreaticoduodenectomy with Roux-en-Y Reconstruction

inaccessible by endoscopy and bypass surgery cannot be performed. In the present study, the obstructed loop was inaccessible by endoscopy due to the Roux-en-Y reconstruction performed in the primary surgery, and bypass surgery was not possible as there was no normal bowel to bypass due to the segmental radiation injury of the afferent loop. Secondly, if endoscopic interventions are unsuccessful in cases of conventional loop reconstruction and bypass surgery cannot be performed. In the Pannala et al. [2] series, 15 (62\%) patients had an endoscopic intervention. These patients required a median of 2 endoscopic procedures (range 1-17 endoscopic procedures); 11 (73\%) patients had clinical and laboratory improvement, 2 (13\%) patients did not improve, and 2 (13\%) patients were lost to follow-up. Patients with local recurrence or multiple areas of stenosis were more likely to require multiple endoscopic interventions and endoscopy was more likely to fail.

\section{Conclusion}

In the present study, the case of a patient with chronic ALO after PD and adjuvant chemoradiation therapy for pancreatic head adenocarcinoma is described. The decisionmaking flow chart of the study patient is presented in fig. 4 . The cause of the obstruction was presumed to be radiation enteritis. The involvement of the afferent loop was segmental and in the length of involvement overlap of radiation enteritis and local recurrence could not be excluded. The Roux-en-Y reconstruction from the primary surgery and the segmental involvement of the afferent loop precluded endoluminal stenting and bypass surgery. Mobilization and removal of the afferent loop was necessary, along with revision of the HJ and PJ.

\section{Disclosure Statement}

None.

\section{References}

1 Fathy 0, Wahab MA, Elghwalby N, Sultan A, EL-Ebidy G, Hak NG, et al: 216 cases of pancreaticoduodenectomy: risk factors for postoperative complications. Hepatogastroenterology 2008;55:1093-1098.

-2 Pannala R, Brandabur JJ, Gan SI, Gluck M, Irani S, Patterson DJ, et al: Afferent limb syndrome and delayed GI problems after pancreaticoduodenectomy for pancreatic cancer: single-center, 14-year experience. Gastrointest Endosc 2011;74:295-302.

-3 Reddy JR, Saxena R, Singh RK, Pottakkat B, Prakash A, Behari A, Gupta K, Kapoor VK: Reoperation following pancreaticoduodenectomy. Int J Surg Oncol 2012;2012:218248.

-4 De Martino C, Caiazzo P, Albano M, Pastore M, Tramutoli PR, Rocca R, Botte M, Sigillito A: Acute afferent loop obstruction treated by endoscopic decompression: case report and review of literature. Ann Ital Chir 2012;83:555-558.

5 Chevallier P, Novellas S, Motamedi JP, Gugenheim J, Brunner P, Bruneton JN: Percutaneous jejunostomy and stent placement for treatment of malignant Roux-en-Y obstruction: a case report. Clin Imaging 2006;30:283-286.

-6 Smith C, Sarr M, vanHeerden J: Completion pancreatectomy following pancreaticoduodenectomy: clinical experience. World J Surg 1992;16:521-524.

7 Macari M, Balthazar EJ: CT of bowel wall thickening: significance and pitfalls of interpretation. AJR Am J Roentgenol 2001;176:1105-1116.

8 Demirjian AN, Kent TS, Callery MP, Vollmer CM: The inconsistent nature of symptomatic pancreaticojejunostomy anastomotic strictures. HPB 2010;12:482-487. 


\section{Case Reports in Oncology}

\begin{tabular}{l|l}
\hline Case Rep Oncol 2013;6:424-429 \\
\hline DOI: 10.1159/000354576 & $\begin{array}{l}\text { ○ 2013 S. Karger AG, Basel } \\
\text { www.karger.com/cro }\end{array}$ \\
\hline
\end{tabular}

Blouhos et al.: Total Reconstruction of the Afferent Loop for Treatment of RadiationInduced Afferent Loop Obstruction with Segmental Involvement after Pancreaticoduodenectomy with Roux-en-Y Reconstruction

9 Morgan KA, Fontenot BB, Harvey NR, Adams DB: Revision of anastomotic stenosis after pancreatic head resection for chronic pancreatitis: is it futile? HPB (Oxford) 2010;12:211-216.

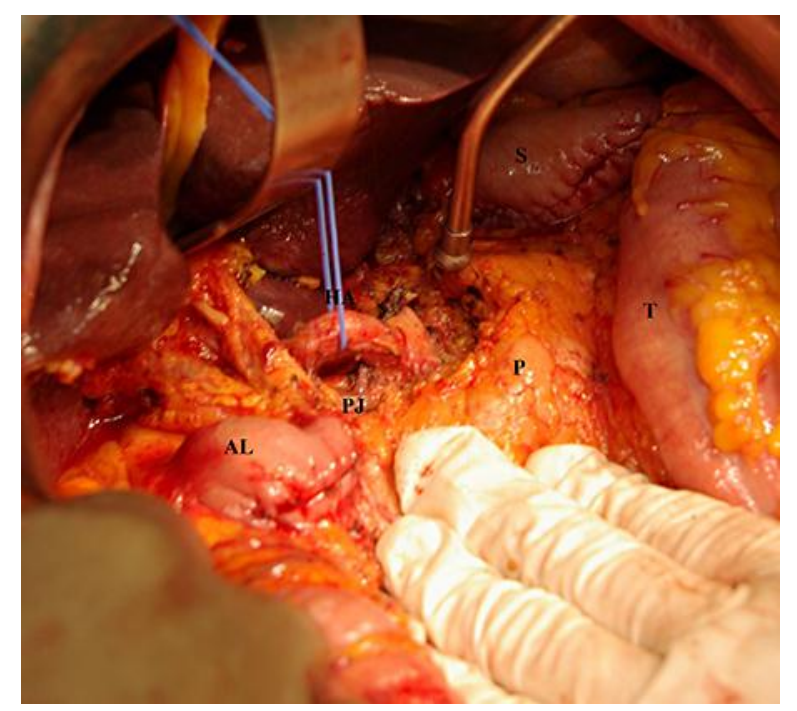

Fig. 1. The post-PD and postradiation therapy surgical field. $\mathrm{S}=$ Gastric remnant; $\mathrm{P}=$ pancreas; $\mathrm{AL}=$ obstructed afferent loop; $\mathrm{HA}=$ hepatic artery; $\mathrm{T}$ = transverse colon.
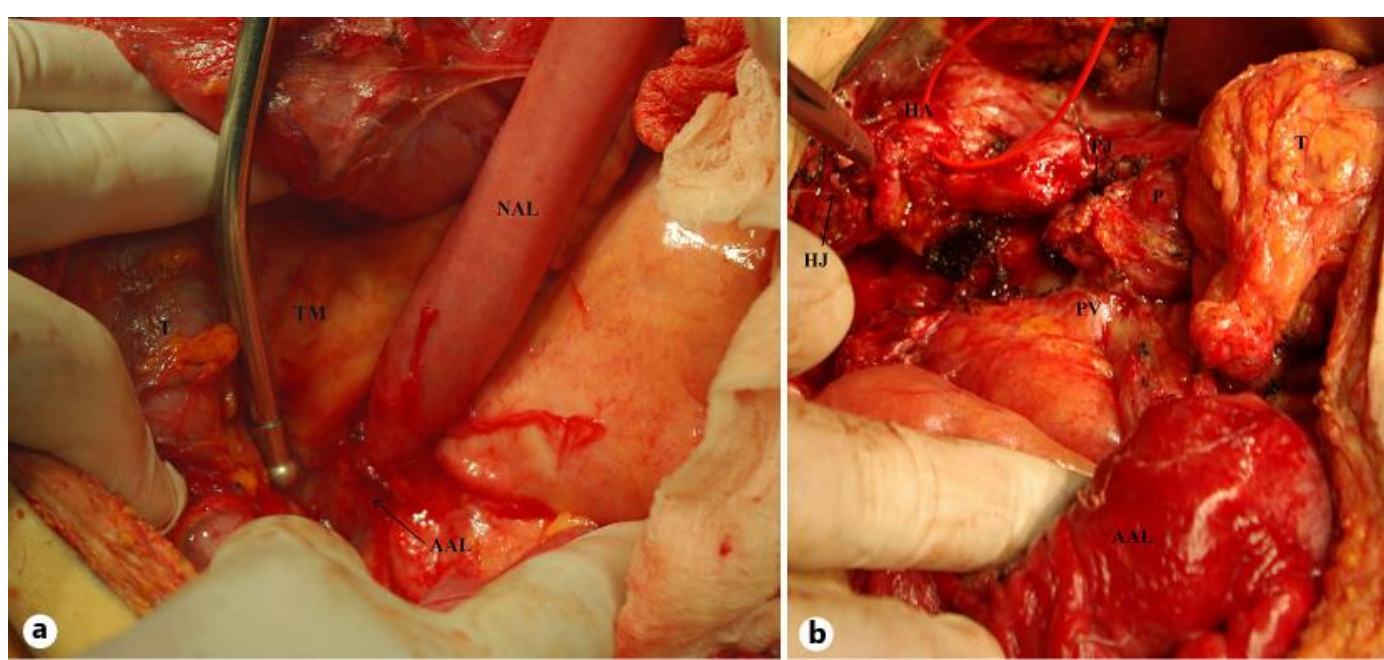

Fig. 2. a Peripherally, the afferent loop was transected distal to the affected segment (AAL). The distal remnant of the normal afferent loop (NAL) was pulled toward the pancreas through the transverse mesocolon (TM) and had a sufficient redundancy to provide construction of the anastomoses without tension. $\mathrm{T}=$ Transverse colon. $\mathbf{b}$ Proximally, the afferent loop was fully mobilized through a field of dense adhesions and scar tissue. The HJ and PJ were taken down and the affected afferent loop (AAL) was removed. The surgical bed of the primary operation was exposed. $\mathrm{P}=$ Pancreas; $\mathrm{HA}=$ hepatic artery; $\mathrm{PV}=$ portal vein; $\mathrm{T}=$ transverse colon. 


\section{Case Reports in Oncology}

\begin{tabular}{l|l}
\hline Case Rep Oncol 2013;6:424-429 \\
\hline DOI: $10.1159 / 000354576$ & $\begin{array}{l}\text { C 2013 S. Karger AG, Basel } \\
\text { www.karger.com/cro }\end{array}$ \\
\hline
\end{tabular}

Blouhos et al.: Total Reconstruction of the Afferent Loop for Treatment of RadiationInduced Afferent Loop Obstruction with Segmental Involvement after Pancreaticoduodenectomy with Roux-en-Y Reconstruction

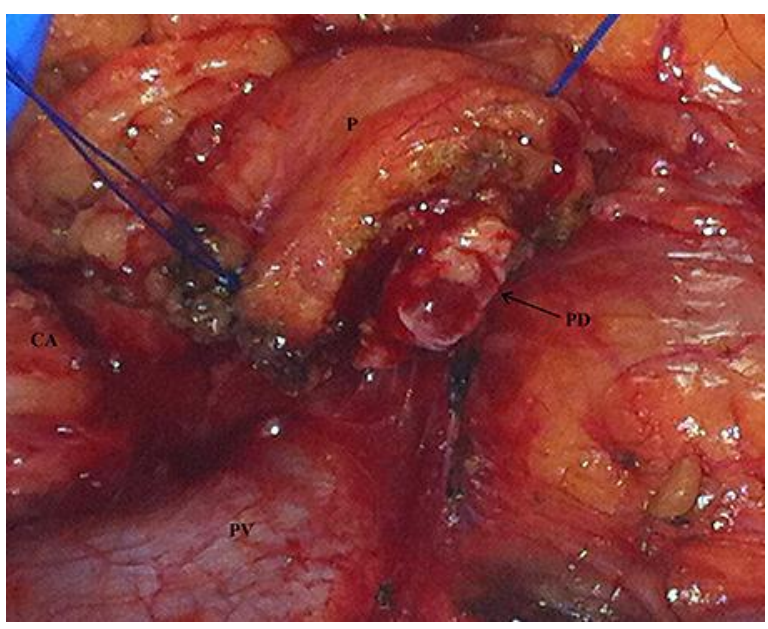

Fig. 3. The PJ was taken down and examined. The remnant pancreas (P) was resected until a dilated and normal pancreatic duct (PD) was exposed. A new duct-to-mucosa PJ was then performed and the reconstruction of the afferent loop was completed after revision of the HJ. CA = Celiac axis; PV = portal vein.

Post PD and radiation therapy patient

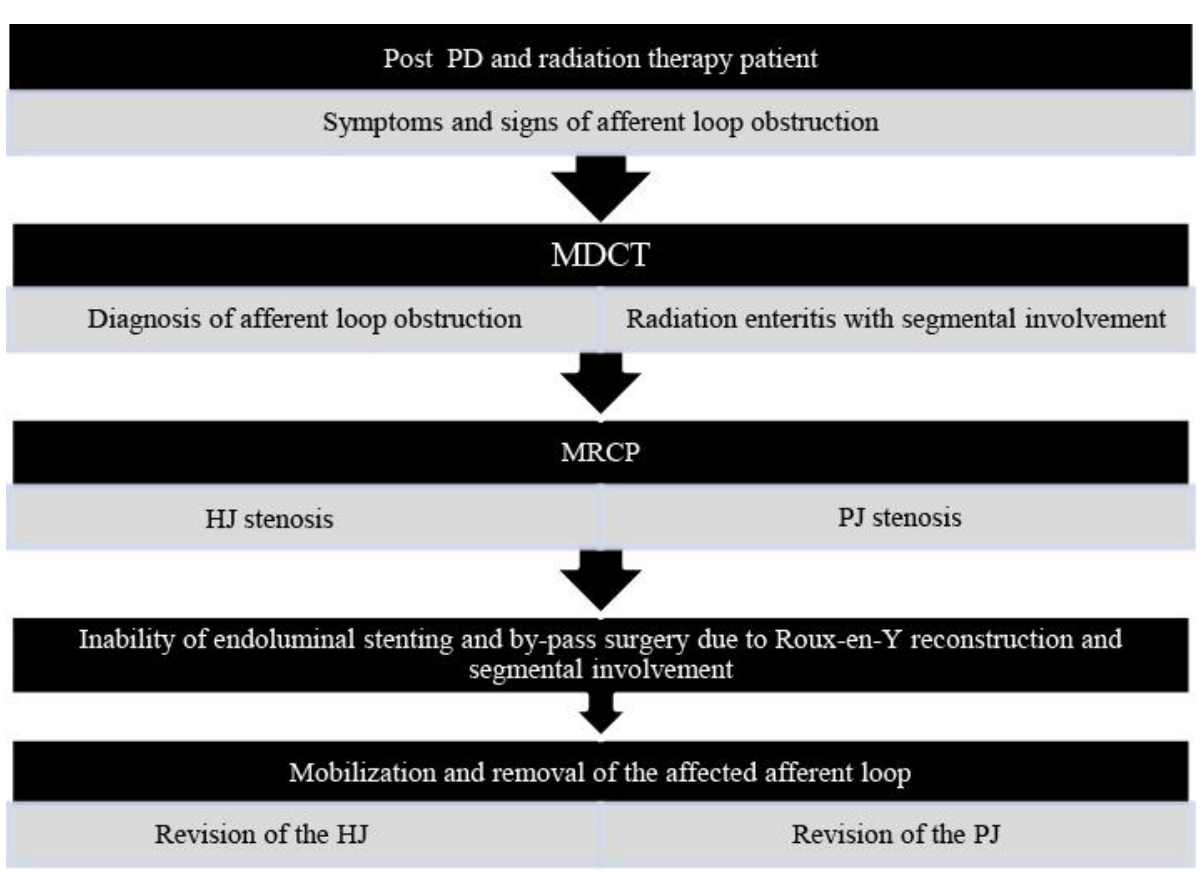

Fig. 4. Decision-making flow chart of the study patient. 